\title{
DIAGONAL SCALING OF STIFFNESS MATRICES IN THE GALERKIN BOUNDARY ELEMENT METHOD
}

\author{
MARK AINSWORTH ${ }^{1}$, BILL MCLEAN $^{2}$ and THANH TRAN ${ }^{3}$
}

(Received 16 March 1998)

\begin{abstract}
A boundary integral equation of the first kind is discretised using Galerkin's method with piecewise-constant trial functions. We show how the condition number of the stiffness matrix depends on the number of degrees of freedom and on the global mesh ratio. We also show that diagonal scaling eliminates the latter dependence. Numerical experiments confirm the theory, and demonstrate that in practical computations involving strong local mesh refinement, diagonal scaling dramatically improves the conditioning of the Galerkin equations.
\end{abstract}

\section{Introduction}

A standard practice in numerical linear algebra is to scale the unknowns and righthand sides of a linear system, with the aim of reducing the condition number of the coefficient matrix. The scaled system will then be less sensitive to roundoff errors; see Forsythe and Moler [4, Chapter 11]. Even if roundoff is not a serious consideration, for instance because discretisation errors dominate, reducing the condition number can speed up the convergence of iterative solvers. Here, we consider a particular class of poorly scaled linear systems, arising in the boundary element method. We derive an estimate for the condition number of the coefficient matrix of such a system, and another, much smaller, estimate for the matrix of an equivalent, appropriately scaled system. Numerical experiments indicate that these estimates are realistic. Our results are related to those of Bank and Scott [2] for domain finite element methods.

Many elliptic boundary value problems can be formulated as integral equations over the boundary of the problem domain. In the boundary element method, such integral

\footnotetext{
'Mathematics Department, Strathclyde University, 26 Richmond St, Glasgow G1 1XH, Scotland.

${ }^{2}$ School of Mathematics, The University of New South Wales, Sydney 2052, Australia.

${ }^{3}$ Centre of Mathematics and its Applications, School of Mathematical Sciences, Australian National University, Canberra 0200, Australia.

(C) Australian Mathematical Society 2000, Serial-fee code 0334-2700/00
} 
equations are solved numerically via a partitioning of the boundary into boundary elements (curvilinear triangles or quadrilaterals, for instance). A good partition should reflect the behaviour of the exact solution of the boundary integral equation. Typically, very small elements are needed in the vicinity of corners or cracks, where the solution becomes singular, but elsewhere, much larger elements suffice. In this way, fewer degrees of freedom are required to achieve a given accuracy than would be the case using a quasi-uniform partition, in which the boundary elements are all of a similar size. Good partitions can be generated automatically by adaptive refinement based on suitable local error indicators, such as those of Carstensen and Stephan [3].

However, the large variation in the sizes of the boundary elements in such a good partition causes the stiffness matrix to be badly scaled, assuming a standard nodal basis is used, and we shall see that the condition number can be much larger than it would be for a quasi-uniform partition with the same number of degrees of freedom. Our goal is to prove that this increase in the condition number is easily eliminated by rescaling the unknowns and right-hand sides in such a way that the diagonal entries of the coefficient matrix are all of the same size. Note that the condition number will still grow as the number of degrees of freedom is increased, unless some additional, more sophisticated preconditioner is used, such as in our earlier work [5].

The paper is organised as follows. Section 2 describes a model problem, involving a weakly singular integral equation of the first kind equivalent to the Dirichlet problem for the Laplacian in three dimensions. Next, Section 3 describes a simple Galerkin discretisation based on piecewise constant trial functions. For the special case of the model problem, our estimates of the condition numbers are given in Theorem 1. Section 4 outlines the proof for a more general case, stated as Theorem 2, and in Section 5 we present the results of some numerical experiments.

Further details have been given in a second paper [1]. There, we also discuss hypersingular boundary integral equations and higher-order boundary elements.

\section{A model problem}

Let $\Omega$ be a bounded Lipschitz domain in $\mathbf{R}^{3}$ and denote the boundary of $\Omega$ by $\Gamma=$ $\partial \Omega$. We consider the Dirichlet problem for the Laplace equation: given boundary data $g$ on $\Gamma$, find $U$ on $\Omega$ satisfying

$$
\Delta U=0 \quad \text { on } \Omega, \quad U=g \text { on } \Gamma .
$$

Let us transform this problem into a boundary integral equation of the first kind. The third Green identity gives, for $x \in \Omega$,

$$
U(x)=\frac{1}{4 \pi} \int_{\Gamma} \frac{1}{|x-y|} \partial_{\nu} U(y) d \sigma_{y}-\frac{1}{4 \pi} \int_{\Gamma} U(y) \frac{\partial}{\partial \nu_{y}}\left(\frac{1}{|x-y|}\right) d \sigma_{y},
$$


where $\nu$ is the outward unit normal to $\Omega$, and $d \sigma$ is the surface element on $\Gamma$. Since $U(y)=g(y)$ is given for $y \in \Gamma$, we can compute $U(x)$ for $x \in \Omega$ once the normal derivative $\partial_{\nu} U$ is known. To this end, take $x$ to be a point on the boundary $\Gamma$, and modify the left-hand side of the third Green identity (1) accordingly, by replacing $U(x)$ with $\frac{1}{2} U(x)$. After rearranging and applying the Dirichlet boundary condition, we find that the unknown $u=\partial_{\nu} U$ satisfies

$$
\frac{1}{4 \pi} \int_{\Gamma} \frac{1}{|x-y|} u(y) d \sigma_{y}=f(x) \quad \text { for } x \in \Gamma,
$$

where

$$
f(x)=\frac{1}{2} g(x)+\frac{1}{4 \pi} \int_{\Gamma} g(y) \frac{v_{y} \cdot(x-y)}{|x-y|^{3}} d \sigma_{y} .
$$

To obtain a numerical solution to (2) using a Galerkin boundary element method, we introduce the symmetric bilinear form

$$
B(u, v)=\frac{1}{4 \pi} \int_{\Gamma} \int_{\Gamma} \frac{u(x) v(y)}{|x-y|} d \sigma_{x} d \sigma_{y}=B(v, u),
$$

and the $L_{2}$ inner product

$$
(f, v)=\int_{\Gamma} f(x) v(x) d \sigma_{x} .
$$

The weak form of (2) can then be written as

$$
B(u, v)=(f, v) \quad \text { for all } v .
$$

The symmetric bilinear form $B$ is bounded on the Sobolev space $H^{-1 / 2}(\Gamma)$, and is also positive and bounded below on this space, that is, there exist positive constants $C$ and $c$ such that

$$
|B(v, w)| \leq C\|v\|_{H^{-1 / 2}(\Gamma)}\|w\|_{H^{-1 / 2}(\Gamma)} \quad \text { and } \quad B(v, v) \geq c\|v\|_{H^{-1 / 2}(\Gamma)}^{2}
$$

for all $v, w \in H^{-1 / 2}(\Gamma)$. Recall that $H^{-1 / 2}(\Gamma)$ is the dual space of $H^{1 / 2}(\Gamma)$, and that a norm for the latter is given by

$$
\|v\|_{H^{1 / 2}(\Gamma)}^{2}=\|v\|_{L_{2}(\Gamma)}^{2}+\int_{\Gamma} \int_{\Gamma} \frac{[v(x)-v(y)]^{2}}{|x-y|^{3}} d \sigma_{x} d \sigma_{y} .
$$

Since $B$ defines an equivalent inner product for $H^{-1 / 2}(\Gamma)$, the Riesz Representation Theorem for Hilbert spaces guarantees the existence of a unique weak solution $u \in$ $H^{-1 / 2}(\Gamma)$ to the boundary integral equation (2). 


\section{The Galerkin boundary element method}

For the simplest possible Galerkin discretisation, we partition the closed surface $\Gamma$ into $N$ boundary elements and define the piecewise constant nodal basis functions

$$
\phi_{k}= \begin{cases}1 & \text { on the } k \text { th element, } \\ 0 & \text { elsewhere on } \Gamma,\end{cases}
$$

for $k=1,2, \ldots, N$. Denoting the trial space by

$$
X=\operatorname{span}\left\{\phi_{1}, \phi_{2}, \ldots, \phi_{N}\right\}
$$

we seek $u_{X} \in X$ satisfying

$$
B\left(u_{X}, v\right)=(f, v) \quad \text { for all } v \in X .
$$

Since $X \subseteq H^{-1 / 2}(\Gamma)$, the existence and uniqueness of $u_{X}$ follow from the properties of $B$ discussed earlier. Furthermore, $u_{X}$ is the best approximation to $u$ from the subspace $X$ if the error is measured in the energy norm, that is,

$$
\left\|u_{X}-u\right\|_{B}=\min _{v \in X}\|v-u\|_{B},
$$

where $\|v\|_{B}=\sqrt{B(v, v)}$. Writing

$$
u_{X}=\sum_{k=1}^{N} \alpha_{k} \phi_{k}
$$

and taking $v=\phi_{j}$ in (4), we obtain the $N \times N$ linear system

$$
\sum_{k=1}^{N} B_{j k} \alpha_{k}=f_{j} \quad \text { for } j=1,2, \ldots, N
$$

where $B_{j k}=B\left(\phi_{j}, \phi_{k}\right)$ and $f_{j}=\left(f, \phi_{j}\right)$. The matrix $B=\left[B_{j k}\right]$ is called the stiffness matrix (with respect to the basis $\left\{\phi_{j}\right\}_{j=1}^{N}$ ). We aim to show that it is better to work with an equivalent, diagonally-scaled system. Thus, let

$$
\widetilde{B}_{j k}=\frac{B_{j k}}{\sqrt{B_{j j} B_{k k}}}=\widetilde{B}_{k j}
$$

and observe that (5) is equivalent to

$$
\sum_{k=1}^{N} \tilde{B}_{j k} \tilde{\alpha}_{k}=\tilde{f_{j}} \quad \text { for } j=1,2, \ldots, N,
$$


where $\tilde{\alpha}_{k}=\sqrt{B_{k k}} \alpha_{k}$ and $\tilde{f_{j}}=f_{j} / \sqrt{B_{j j}}$. Both the coefficient matrices $B$ and $\widetilde{B}$ are symmetric and positive-definite, but whereas $\widetilde{B}_{j j}=1$ for all $j$, the diagonal entries of $B$ can vary greatly in size if some boundary elements are much smaller than others. Notice that if we put

$$
\tilde{\phi}_{k}=\frac{\phi_{k}}{\sqrt{B_{k k}}}=\frac{\phi_{k}}{\left\|\phi_{k}\right\|_{B}}
$$

so that $\left\|\tilde{\phi}_{k}\right\|_{B}=1$, then

$$
u_{X}=\sum_{k=1}^{N} \tilde{\alpha}_{k} \tilde{\phi}_{k}
$$

Thus, diagonal scaling of the linear system is equivalent to normalising the nodal basis functions with respect to the energy norm. Letting $h_{k}$ denote the diameter of the $k$ th boundary element, and putting $h_{\max }=\max _{1 \leq k \leq N} h_{k}$ and $h_{\min }=\min _{1 \leq k \leq N} h_{k}$, we state a special case of our main result as Theorem 1 below. The proof requires the partition to be non-degenerate, that is, the ratio of the diameter of an element to the diameter of the largest inscribed ball must be uniformly bounded. It follows that the partition is locally quasi-uniform, but the global mesh ratio $h_{\max } / h_{\min }$ can still be arbitrarily large.

THEOREM 1. Consider the piecewise-constant Galerkin boundary element method applied to the three-dimensional, harmonic single-layer potential equation (2). If the partition is non-degenerate, then the $\ell_{2}$ condition number of the stiffness matrix satisfies

$$
\operatorname{cond}(B) \leq C N^{1 / 2}\left(h_{\max } / h_{\min }\right)^{3},
$$

whereas for the diagonally scaled matrix,

$$
\operatorname{cond}(\widetilde{B}) \leq C N^{1 / 2}
$$

\section{Outline of the proof}

Recall that if $B$ is any real, symmetric $N \times N$ matrix, and if there are positive constants $\lambda$ and $\Lambda$ such that

$$
\lambda|\beta|^{2} \leq \beta^{T} B \beta \leq \Lambda|\beta|^{2} \quad \text { for all } \beta \in \mathbf{R}^{N},
$$

then every eigenvalue of $B$ lies in the closed interval $[\lambda, \Lambda]$. Since the $\ell_{2}$ condition number equals the ratio of the largest to the smallest eigenvalue, one obtains the upper bound

$$
\operatorname{cond}(B) \leq \Lambda / \lambda
$$


In proving Theorem 1 , we may as well generalise the setting by assuming simply that $B$ is a symmetric bilinear form, bounded and positive bounded below on $H^{-m}(\Gamma)$, for a real number $m$ satisfying

$$
0<2 m<d=\text { dimension of } \Gamma
$$

in our model problem, $m=1 / 2$ and $d=2$. Denote the $k$ th boundary element by $\Gamma_{k}$, so that

$$
\Gamma_{k}=\text { support of } \phi_{k} \text {, }
$$

and consider an arbitrary piecewise-constant function

$$
v=\sum_{k=1}^{N} v_{k} \quad \text { with } \quad v_{k}=\beta_{k} \phi_{k}
$$

The desired estimates of $\operatorname{cond}(B)$ and $\operatorname{cond}(\widetilde{B})$ will follow with the help of three technical lemmas stated below. We omit the proofs of the first two, but point out that the norm in $H^{-m}\left(\Gamma_{k}\right)$ must be defined carefully in order for Lemmas 1 and 2 to be valid, because some of the imbedding constants for the various standard equivalent norms depend on the diameter of $\Gamma_{k}$. Also, when $d=1$ the non-degeneracy assumption on the partition must be strengthened by explicitly requiring local quasi-uniformity. For further details, see [1].

LEMMA 1. $\left\|\phi_{k}\right\|_{H^{-m}(\Gamma)}^{2} \simeq h_{k}^{d+2 m} \simeq\left\|\phi_{k}\right\|_{H^{-m}\left(\Gamma_{k}\right)}^{2}$.

LEMMA 2. $\sum_{k=1}^{N}\left\|v_{k}\right\|_{H^{-m}\left(\Gamma_{k}\right)}^{2} \leq C\|v\|_{H^{-m}(\Gamma)}^{2}$.

LEMMA 3. $\|v\|_{H^{-m}(\Gamma)}^{2} \leq C N^{2 m / d} \sum_{k=1}^{N}\left\|v_{k}\right\|_{H^{-m}(\Gamma)}^{2}$

PROOF. The Sobolev imbedding theorem gives $H^{m}(\Gamma) \subseteq L_{p^{*}}(\Gamma)$ for $p^{*}=2 d /(d-$ $2 m$ ), so by duality,

$$
\|v\|_{H^{-m}(\Gamma)} \leq C\|v\|_{L_{\rho}(\Gamma)} \quad \text { for } \quad p=\frac{2 d}{d+2 m}
$$

and here $1<p<2$ because of our assumption that $0<2 m<d$. Let $q=2 / p$ and note that

$$
1<q<2 \text { and } \frac{1}{q^{*}}=1-\frac{1}{q}=1-\frac{p}{2}
$$


so by Hölder's inequality,

$$
\begin{aligned}
\|v\|_{L_{p}(\Gamma)}^{p} & =\sum_{k=1}^{N}\left\|v_{k}\right\|_{L_{\rho}(\Gamma)}^{p} \leq\left(\sum_{k=1}^{N} 1\right)^{1 / q^{*}}\left(\sum_{k=1}^{N}\left\|v_{k}\right\|_{L_{p}(\Gamma)}^{p q}\right)^{1 / q} \\
& =N^{1-p / 2}\left(\sum_{k=1}^{N}\left\|v_{k}\right\|_{L_{p}(\Gamma)}^{2}\right)^{1 / q} .
\end{aligned}
$$

Since $\left\|\phi_{k}\right\|_{L_{p}(\Gamma)}^{2} \leq C h_{k}^{2 d / p}=C h_{k}^{d+2 m} \leq C\left\|\phi_{k}\right\|_{H^{-m}(\Gamma)}^{2}$, we have

$$
\begin{aligned}
\|v\|_{H^{-m}(\Gamma)}^{2} & \leq C\left(\|v\|_{L_{p}(\Gamma)}^{p}\right)^{2 / p} \leq C N^{2 / p-1} \sum_{k=1}^{N}\left\|v_{k}\right\|_{L_{p}(\Gamma)}^{2} \\
& \leq C N^{2 m / d} \sum_{k=1}^{N}\left\|v_{k}\right\|_{H^{-m}(\Gamma)}^{2} .
\end{aligned}
$$

Theorem 1 now follows by taking $\dot{m}=1 / 2$ and $d=2$ in the next result. We point out that the restriction to piecewise-constants is not essential. The method of proof is readily extended to treat higher-order boundary element methods; see [1].

THEOREM 2. Let $0<2 m<d$ and assume that $\Gamma$ is the $d$-dimensional boundary of a domain in $\mathbf{R}^{d+1}$. Consider the piecewise-constant Galerkin method applied to a variational problem (3) with a symmetric bilinear form $B$ satisfying

$$
B(v, v) \simeq\|v\|_{H^{-m}(\Gamma)}^{2} \quad \text { for all } v \in H^{-m}(\Gamma) .
$$

If the partition is non-degenerate, then the $\ell_{2}$ condition number of the stiffness matrix satisfies

$$
\operatorname{cond}(B) \leq C N^{2 m / d}\left(h_{\max } / h_{\min }\right)^{d+2 m},
$$

whereas for the diagonally scaled matrix,

$$
\operatorname{cond}(\widetilde{B}) \leq C N^{2 m / d} .
$$

PROOF. If the column vector $\beta \in \mathbf{R}^{N}$ is as in (6), then

$$
\beta^{T} B \beta=B(v, v) \simeq\|v\|_{H^{-m}(\Gamma)}^{2},
$$

and by Lemma 1 ,

$$
\left\|v_{k}\right\|_{H^{-m}(\Gamma)}^{2}=\beta_{k}^{2}\left\|\phi_{k}\right\|_{H^{-m}(\Gamma)}^{2} \simeq \beta_{k}^{2} h_{k}^{d+2 m}
$$


Thus, Lemmas 2 and 3 give

$$
c \sum_{k=1}^{N} \beta_{k}^{2} h_{k}^{d+2 m} \leq \beta^{T} B \beta \leq C N^{2 m / d} \sum_{k=1}^{N} \beta_{k}^{2} h_{k}^{d+2 m},
$$

so

$$
c h_{\min }^{d+2 m}|\beta|^{2} \leq \beta^{T} B \beta \leq C N^{2 m / d} h_{\max }^{d+2 m}|\beta|^{2},
$$

and the bound for $\operatorname{cond}(B)$ follows. However, if we put

$$
\tilde{v}=\sum_{k=1}^{N} \tilde{v}_{k}, \quad \tilde{v}_{k}=\beta_{k} \tilde{\phi}_{k} \quad \text { and } \quad\left\|\tilde{\phi}_{k}\right\|_{H^{-m}(\Gamma)}^{2} \simeq 1,
$$

then

$$
\beta^{T} \tilde{B} \beta=B(\tilde{v}, \tilde{v}) \simeq\|\tilde{v}\|_{H^{-m}(\Gamma)}^{2},
$$

and hence

$$
c|\beta|^{2} \leq \beta^{T} \widetilde{B} \beta \leq C N^{2 m / d}|\beta|^{2},
$$

giving the bound for $\operatorname{cond}(\widetilde{B})$.

In [1], we consider also the limiting case when $d=2 m$, and show that the estimates of Theorem 2 remain valid if certain logarithmic factors are inserted. This case occurs with $m=1 / 2$ and $d=1$, when the Dirichlet problem for the Laplace equation in $t w o$ dimensions is solved via the usual first-kind boundary integral formulation.

\section{Numerical experiments}

To test whether our theoretical upper bounds for the condition numbers were realistic, we performed some simple experiments with two test problems.

For the first test problem, we considered the integral equation (2) with $\Gamma$ a square plate in $\mathbf{R}^{3}$, and used quadrilateral elements. The theory as given above does not cover this case, because $\Gamma$ is an open surface, but our arguments can be modified to show that the conclusions of Theorem 1 still hold. The numerical results are shown in Table 1. As well as giving the values of the $\ell_{2}$ condition numbers, we show in parentheses the inferred values of the exponent $r$ such that the condition number is asymptotically proportional to $N^{r}$. As expected, cond $(B)=O\left(N^{1 / 2}\right)$ for a uniform partition of the type shown in Figure 1 (a). Table 1 also gives the condition numbers of $B$ and of the diagonally scaled matrix $\widetilde{B}$ for a graded partition 


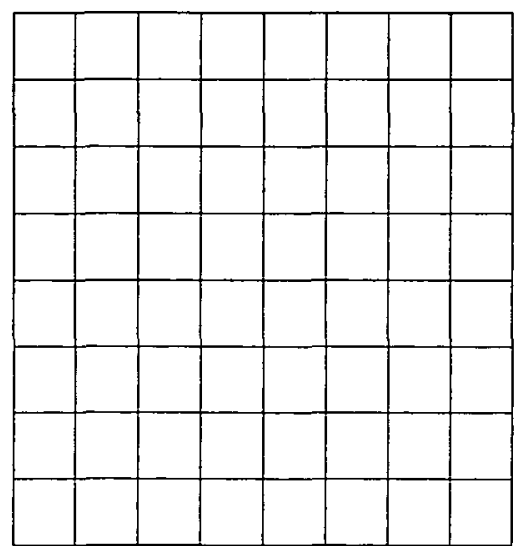

(a) Uniform partition

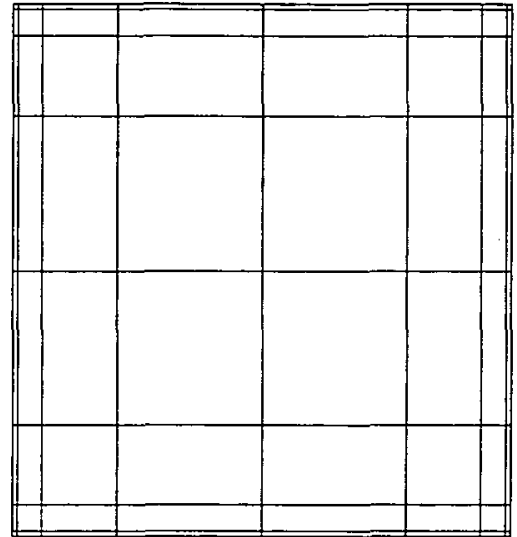

(b) Graded partition

FIGURE 1. Uniform and graded partitions for a square plate in $\mathbf{R}^{3}$.

TABLE 1. Condition numbers for the first test problem (square plate in $\mathbf{R}^{3}$ ).

\begin{tabular}{|r|cl|ll|ll|}
\hline & \multicolumn{2}{|c|}{ Uniform Partition } & \multicolumn{4}{c|}{ Graded Partition } \\
$N$ & $\operatorname{cond}(B)$ & \multicolumn{2}{c|}{$\operatorname{cond}(B)$} & $0.3324 \mathrm{E}+01$ \\
& $0.3324 \mathrm{E}+01$ & & $0.3324 \mathrm{E}+01$ & & 0. \\
16 & $0.7750 \mathrm{E}+01$ & $(0.61)$ & $0.7148 \mathrm{E}+03$ & $(3.87)$ & $0.7079 \mathrm{E}+01$ & $(0.55)$ \\
64 & $0.1635 \mathrm{E}+02$ & $(0.54)$ & $0.1268 \mathrm{E}+06$ & $(3.74)$ & $0.1613 \mathrm{E}+02$ & $(0.59)$ \\
256 & $0.3323 \mathrm{E}+02$ & $(0.51)$ & $0.1764 \mathrm{E}+08$ & $(3.56)$ & $0.4427 \mathrm{E}+02$ & $(0.73)$ \\
1024 & $0.6678 \mathrm{E}+02$ & $(0.50)$ & $0.2304 \mathrm{E}+10$ & $(3.51)$ & $0.1158 \mathrm{E}+03$ & $(0.69)$ \\
\hline
\end{tabular}

of the type shown in Figure 1 (b). As $N \rightarrow \infty$, the maximum and minimum element diameters behave like $h_{\max } \simeq N^{-1}$ and $h_{\min } \simeq N^{-2}$. Although the partition includes degenerate elements, we nevertheless find that $\operatorname{cond}(B)=O\left(N^{7 / 2}\right)$, and that $\operatorname{cond}(\widetilde{B})$ is something like $O\left(N^{1 / 2}\right)$, in accordance with the estimates of Theorem 1 .

The second test problem was the analogue of (2) for the Dirichlet problem in $\mathbf{R}^{2}$,

$$
\frac{1}{2 \pi} \int_{\Gamma} \log \left(\frac{5}{|x-y|}\right) u(y) d \sigma_{y}=f(x) \quad \text { for } x \in \Gamma .
$$

For $\Gamma$, we chose the boundary of the L-shaped polygon with vertices $(0,0),(0,1)$, $(-1,1),(-1,-1),(1,-1)$ and $(1,0)$. Table 2 sets out our numerical results. For a uniform partition of the type shown in Figure 2 (a), we observe that the condition number is $O(N)$, in agreement with Theorem 2 and the remark following its proof. For a graded mesh of the type shown in Figure $2(\mathrm{~b})$, with $h_{\max } \simeq N^{-1}$ and $h_{\min } \simeq N^{-3}$, we expect $\operatorname{cond}(B)=O\left(N^{5}\right)$ and $\operatorname{cond}(\widetilde{B})=O(N)$, ignoring some logarithmic factors, and the numerical results are again consistent with our theoretical bounds. 


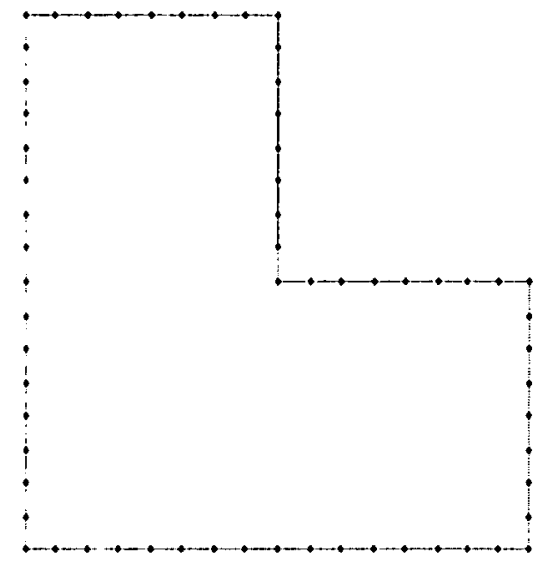

(a) Uniform partition

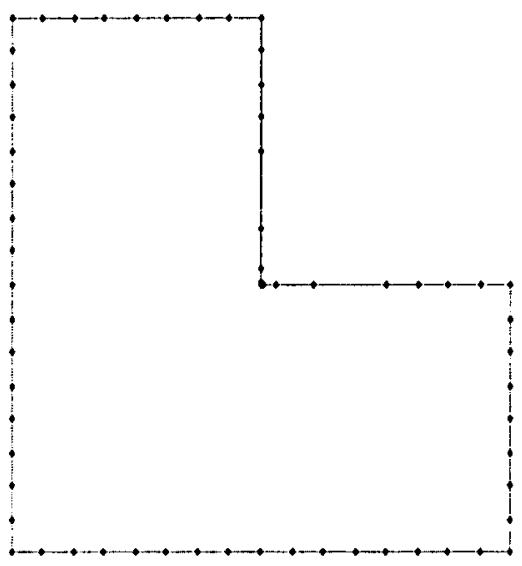

(b) Graded partition

FIGURE 2. Uniform and graded partitions for an L-shaped polygon in $\mathbf{R}^{2}$.

TABLE 2. Condition numbers for the second test problem (polygon in $\mathbf{R}^{2}$ ).

\begin{tabular}{|r|cc|cc|cc|}
\hline & \multicolumn{2}{|c|}{ Uniform Partition } & \multicolumn{4}{c|}{ Graded Partition } \\
$N$ & \multicolumn{2}{|c|}{$\operatorname{cond}(B)$} & \multicolumn{2}{c|}{$\operatorname{cond}(B)$} & \multicolumn{2}{c|}{$\operatorname{cond}(\widetilde{B})$} \\
\hline 8 & $0.1773 \mathrm{E}+02$ & & $0.4040 \mathrm{E}+02$ & & $0.1539 \mathrm{E}+02$ & \\
16 & $0.3290 \mathrm{E}+02$ & $(0.89)$ & $0.3290 \mathrm{E}+02$ & $(-0.30)$ & $0.3290 \mathrm{E}+02$ & $(1.10)$ \\
32 & $0.6297 \mathrm{E}+02$ & $(0.94)$ & $0.8009 \mathrm{E}+03$ & $(4.61)$ & $0.6398 \mathrm{E}+02$ & $(0.96)$ \\
64 & $0.1233 \mathrm{E}+03$ & $(0.97)$ & $0.2633 \mathrm{E}+05$ & $(5.04)$ & $0.1467 \mathrm{E}+03$ & $(1.20)$ \\
128 & $0.2450 \mathrm{E}+03$ & $(0.99)$ & $0.8485 \mathrm{E}+06$ & $(5.01)$ & $0.3306 \mathrm{E}+03$ & $(1.17)$ \\
256 & $0.4897 \mathrm{E}+03$ & $(1.00)$ & $0.2720 \mathrm{E}+08$ & $(5.00)$ & $0.7227 \mathrm{E}+03$ & $(1.13)$ \\
512 & $0.9793 \mathrm{E}+03$ & $(1.00)$ & $0.8708 \mathrm{E}+09$ & $(5.00)$ & $0.1549 \mathrm{E}+04$ & $(1.10)$ \\
\hline
\end{tabular}

\section{References}

[1] M. Ainsworth, W. McLean and T. Tran, "The conditioning of boundary element equations on locally refined meshes and preconditioning by diagonal scaling", SIAM J. Numer. Anal. 36 (1999) 1901-1932.

[2] R. E. Bank and L. R. Scott, "On the conditioning of finite element equations with highly refined meshes", SIAM J. Numer. Anal. 26 (1989) 1383-1394.

[3] C. Carstensen and E. P. Stephan, "A posteriori error estimates for boundary element methods", Math. Comp. 64 (1995) 483-500.

[4] G. E. Forsythe and C. B. Moler, Computer Solution of Linear Algebraic Systems (Prentice-Hall, 1967).

[5] W. McLean and T. Tran, “A preconditioning strategy for boundary element Galerkin methods", Numer. Methods Partial Differential Eq. 13 (1997) 283-301. 